\title{
OPTIMAL PROCESS PARAMETERS SELECTION OF LASER TRANSMISSION WELDING OF POLYCARBONATE USING CUCKOO SEARCH ALGORITHM
}

\section{OPTIMIZATION OF LASER TRANSMISSION WELDING PROCESS USING CUCKOO SEARCH ALGORITHM}

\begin{tabular}{|c|c|c|c|}
\hline Bappa Acherjee & Arunanshu S. Kuar & Debanjan Maity & Souren Mitra \\
Production Engineering & Production Engineering & Production Engineering & Production Engineering \\
Department, Birla Institute of & Department, & Department, & Department, \\
Technology: Mesra, & Jadavpur University, Kolkata & Jadavpur University, & Jadavpur University, \\
Deoghar Campus, & -700032 , India & Kolkata - 700032, India & Kolkata - 700032, India \\
$\begin{array}{c}\text { Deoghar-814142, India } \\
\text { bappa.rana@gmail.com, }\end{array}$ & & & \\
a.bappa@yahoo.com & & & \\
\hline
\end{tabular}

\begin{abstract}
In present work laser transmission welding of polycarbonate is investigated and optimized using cuckoo search algorithm (CSA) incorporation with response surface methodology (RSM). Objectives are set to obtain higher weld strength as well as lower weld width to achieve better product quality. Parametric optimizations are carried out using CSA, in which the objective functions are developed using RSM. Results show that CSA gives comparatively better results than conventional optimization techniques. This is because of the fact that CSA can locate the global optima, as no parameters are held constant during the optimization process. This algorithm is also found to be capable of predicting accurate trends of the parametric effects of laser transmission welding of
\end{abstract} polycarbonate.

Keywords-laser transmission welding; cuckoo search algorithm; metaheuristic algorithm; RSM; optimization

\section{INTRODUCTION}

The primary and main advantages of using plastic components are due to their high strength-to-weight ratio, lower cost of manufacturing, good impact strength, and noncorrosive property. Military and commercial aircraft canopies are now manufactured from polycarbonate, which are significantly lighter than glass and many times also have impact strength better than glass. Polycarbonate is also used for window dust covers, lenses, displays, cabinets and shades in the aircraft.

The basic principle of laser transmission welding (LTW) is to create a reliable joint between two overlapping thermoplastic parts by transmitting a laser beam through a laser-transparent part and welding at the interface with the laser-absorbing part [1]. Unlike the standard welding processes where the energy is applied at the surface of the materials, laser transmission welding aims to apply the energy in between two plastic pieces at their interface. The process is non contact, non contaminant and flexible. It shows excellent integration capabilities and potential for automation. There is an absence of vibration of the parts involved in the process, and it introduces minimal thermal stress and avoids particle generation. LTW process is thus preferred over its conventional counterparts, and has found several state-of-theart applications in microfluidics, micro electro-mechanical systems (MEMS) and biomedicine [2].

During laser transmission welding, a laser beam is focused at two overlapping thermoplastic parts of different optical properties. The top of which is to be transparent to the radiation at the laser wavelength, and the other is to be absorbent to that radiation. Carbon black pigments of different proportions are mixed with thermoplastics to render it laser absorbing. The transmitted energy is absorbed over a certain depth of that material and converted to heat which depends on the thickness and absorption coefficient of the absorbing part. The generated heat is transported by thermal conduction into the top transparent part and also to the deeper layers of absorbing part. Consequently, both the parts melt at the joining interface, and a solid joint is formed as the melt layer solidifies [3]. After cooling and resolidification, a bond is formed at the weld seam [4].

The most important independent process parameters for the laser transmission welding are laser power, welding speed, size of the laser beam spot on the work-piece and clamping pressure [5]. These key control parameters successively can control the temperature field inside the weld seam hence also the weld quality. Baylis et al. [6] investigated the effect of laser welding parameters on the laser transmission weld quality, characterized by weld width and strength for lap welded thermoplastic elastomers to polypropylene. Acherjee et al. [7] established a correlation among the LTW parameters and output variables through a nonlinear model developed by artificial neural network. The parametric influence on the model outputs was also investigated through sensitivity analysis. A number of research works have been performed to investigate the effect of process parameters on weld quality [89]. Several attempts have also been made to select the optimal process parameters to achieve the desired weld quality [10$11]$.

Cuckoo search algorithm (CSA) is a recent development in the field of swarm intelligence population based metaheuristic optimization algorithm inspired from the curious breeding behavior of cuckoo birds. Previous researches [12-13] have effectively shown that cuckoo search algorithm can effectively outperform the existing algorithms such as genetic algorithm and particle swarm optimization which motivates the present study. In this paper, the objective is set to search for the optimal process widow for laser transmission welding of polycarbonates, to achieve desired product quality. Experimental scheme is planned using central composite rotatable design of RSM. Empirical models developed by using RSM are used as objective functions for optimization using CSA. Results are further compared with the optimum results found with conventional optimization techniques. In addition to that parametric trends are also analyzed. 


\section{METHODOLOGY}

\section{A. Response surface methodology}

Response surface methodology is a collection of mathematical and statistical techniques that are useful for the modeling and analysis of problems in which a response of interest is influenced by several variables and the objective is to optimize the response [14]. In practical applications of RSM, it is necessary to develop an approximating model for the true response surface. The approximating model is based on observed data from the process or system and is an empirical model. Multiple regression is a collection of statistical techniques useful for building the types of empirical models required in RSM. Usually, a second-order polynomial equation is used in RSM.

\section{B. Cuckoo search algorithm}

The cuckoo search algorithm is swarm intelligence based meta-heuristic algorithm, proposed by Yang and Deb [15, 16]. The algorithm is inspired from curious breeding behavior of cuckoo birds. Cuckoo birds lay their eggs in the nests of other host birds (almost other species) with amazing abilities such as selecting the recently spawned nests and removing existing eggs that increase hatching probability of their eggs. Cuckoos engage brood parasitism. It is a type of parasitism in which a bird (brood parasite) lays and abandons its eggs in the nest of another species. Some of host birds are able to combat this parasite behavior of cuckoos and throw out the discovered alien eggs or build their new nests in new locations. In other situations, more friendly hosts will simply abandon its nest and build a new nest elsewhere. The basic concept of CSA has been described shortly below:

Each egg in a nest represents a solution and a cuckoo egg represents a new one. If the cuckoo egg is very similar to the host's, then this cuckoo egg is less likely to be discovered; thus, the fitness should be related to the difference in solutions. The aim is to employ the new and potentially better solutions (cuckoos) to replace a not-so-good solution in the nests. For better understanding there are three idealized rules, which are mentioned below [15]:

- Each cuckoo lays one egg at a time and dumps it in a randomly chosen nest.

- The best nests with high-quality eggs will be carried over to the next generations.

- The number of available host nests is fixed, and the egg laid by a cuckoo is discovered by the host bird with a probability $P_{a} \in[0,1]$. In this case, the host bird can either get rid of the egg or simply abandon the nest and build a completely new nest.

The last assumption can be approximated by the fraction $P a$ of the $n$ nests which are replaced by new nests (with new random solutions).

A l'evy flight is performed when new solutions $x_{i}^{t+1}$ are generated for say cuckoo $i$, as given below [15]:

$$
x_{i}^{(t+1)}=x_{i}^{t}+\alpha \text { a L'evy }(\gamma)
$$

where, $\alpha>0$ is the step size which should be related to the scales of the problem of interest. In most cases, we can use $\alpha$ $=\mathrm{O}(1)$. The product $(\mathrm{a})$ means entry-wise multiplications. L'evy flights essentially provide a random walk while their random steps are drawn from an L'evy distribution for large steps, as [15]:

$$
L^{\prime} \text { evy } \sim \mathrm{u}=\mathrm{t}^{-\gamma}(1<\gamma<3)
$$

which has an infinite mean as well as infinite variance. Here the consecutive jumps/steps of a cuckoo essentially form a random walk process which obeys a power-law step-length distribution with a heavy tail.

\section{EXPERIMENTAL WORK}

Injection moulded natural and black polycarbonate plaques of dimensions $70 \mathrm{~mm} \times 35 \mathrm{~mm} \times 4 \mathrm{~mm}$, each, are used as the work-materials, as shown in Fig. 1. Laser welding is performed with a Coherent ${ }^{\circledR}$ FAP continuous wave diode laser system. A three-axis motion system is used for the experimental work. The workpieces are moved via a CNC X$\mathrm{Y}$ table, coordinated with the motion system and computer interface. Experimental scheme is planned using four-factors five-levels central composite rotatable design of RSM. Four key factors namely laser power, welding speed, stand-off distance and clamp pressure are considered as input process parameters. Their symbols, units and ranges are given in Table I. The welding parameters are of different magnitude and order, thus, the parameters are normalized and each parameter is constrained within the values corresponding to -2 and +2 coded levels. The two responses recorded are weld strength and weld width. Weld strength of each specimen is measured using a microprocessor controlled Instron ${ }^{\circledR}$ universal testing machine. The shear-lap joints are tested at a crosshead speed of $0.5 \mathrm{~mm} / \mathrm{min}$. The weld strength is calculated as the maximum load to failure per unit length of the weld $(\mathrm{N} / \mathrm{mm})$. An Olympus ${ }^{\circledR}$ STM 6 measuring microscope is used for measuring weld seam widths. The STM6 microscope offers high performance three-axis measurements of parts, with submicron precision.

TABLE I. CONTROL PARAMETERS AND THEIR CODED LEVELS [11]

\begin{tabular}{|c|c|c|c|c|c|c|c|}
\hline Parameters & \multirow{2}{*}{ Unit } & Symbol & \multicolumn{5}{|c|}{ Levels } \\
\cline { 4 - 8 } & & & $-\mathbf{2}$ & $-\mathbf{1}$ & $\mathbf{0}$ & $\boldsymbol{1}$ & $\mathbf{2}$ \\
\hline Laser power & $\mathrm{W}$ & $\mathrm{P}$ & 10 & 12.5 & 15 & 17.5 & 20 \\
\hline Welding speed & $\mathrm{mm} / \mathrm{s}$ & $\mathrm{S}$ & 4 & 7 & 10 & 13 & 16 \\
\hline Standoff distance & $\mathrm{mm}$ & $\mathrm{F}$ & 25 & 28 & 31 & 34 & 37 \\
\hline Clamp pressure & $\mathrm{MPa}$ & $\mathrm{C}$ & 1.2 & 2.4 & 3.6 & 4.8 & 6.0 \\
\hline
\end{tabular}

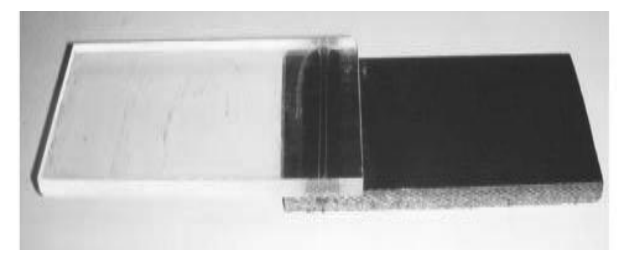

Fig. 1. Welded specimen

\section{DEVELOPMENT OF EMPIRICAL MODELS USING RSM}

Response surface methodology is employed to develop the mathematical models, which can be used to predict the weld strength and weld width for laser transmission welding of polycarbonates. The adequacy of the models is tested using the sequential $F$-test, lack-of-fit test and the analysis-of- 


\section{Asia Pacific Journals}

variance technique (ANOVA) to obtain the best-fit models. The fit summary for weld strength and weld width, both, suggest the quadratic models where the additional terms are significant and the models are not aliased. Further it is observed that the adequacy measures $R^{2}$, adjusted $R^{2}$ and predicted $R^{2}$ are in reasonable agreement and are close to 1 , which indicate adequacy of the models. The adequate precision compares the signal to noise ratio and a ratio greater than 4 is desirable. The value of adequate precision ratio for both the models are greater than the prescribed value which indicate adequate model discrimination. The lack-of-fit $F$ values for weld strength and weld width models are found to be not significant relative to the pure error. The models developed in terms of coded factors using RSM, determined by Design expert ${ }^{\circledR}$ software, are given below:

Weld strength, $W S(\mathrm{~N} / \mathrm{mm})=51.21+7.05 P-6.33 S+6.87 F$ $+0.030 C+0.90 P S+2.28 P F$ $-2.04 S F-1.22 S^{2}-1.16 F^{2}-$ $1.24 C^{2}$

Weld width, $W W(\mathrm{~mm})=2.83+0.10 P-0.33 S+0.32 F-$ 2.917E-003 $C-0.044 P S+0.048$ $P C-0.043 S C-0.036 A^{2}+$ $0.048 B^{2}+0.050 C^{2}$

Fig. 2 presents the relationship between the actual and predicted values of weld strength and weld width. These figures indicates that the developed models for weld strength and weld width are adequate and predicted results are in good agreement with measured data.
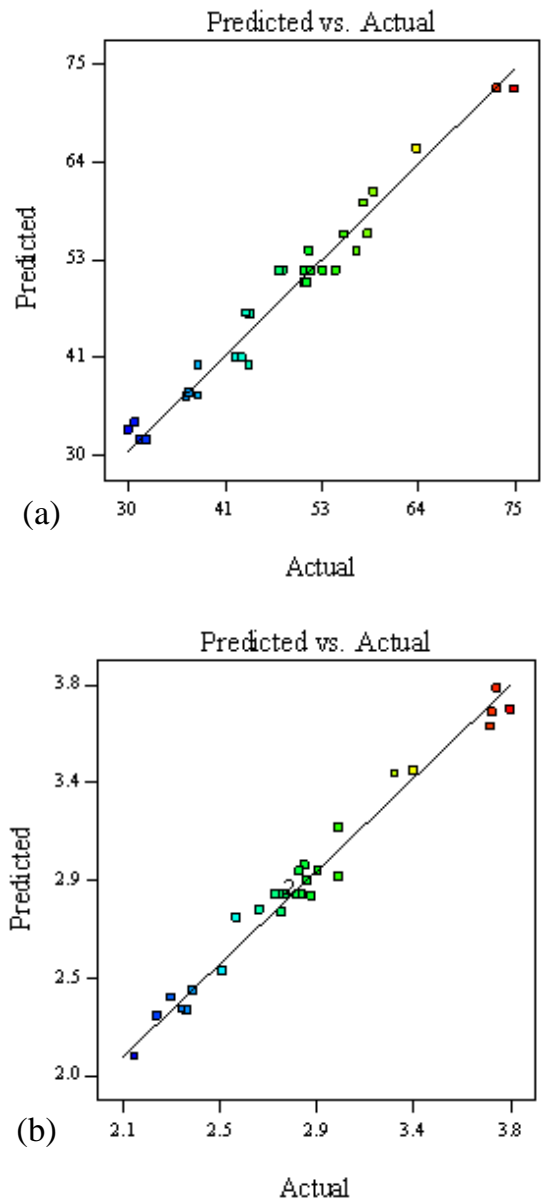

Fig. 2. Plot of actual vs. predicted response of (a) weld strength results, and (b) weld width results
ELK Asia Pacific Journals - Special Issue

ISBN: 978-81-930411-8-5

\section{OPTIMIZATION USING CUCKOO SEARCH ALGORITHM}

In laser transmission welding, the weld strength is higher the better criteria, whereas, weld width is lower the better criteria. Thus, the optimization criteria are set to achieve maximum weld strength and minimum weld width, to obtain desired weld quality. Cuckoo search algorithm is utilized to find the optimal set of LTW parameters. For this, a computer program is developed in MATLAB ${ }^{\circledR}$ on an Intel ${ }^{\circledR}$ Core $^{\mathrm{TM}}$ i3380M CPU@2.53 GHz, 3.00 GB RAM operating platform based on the pseudo code of cuckoo search algorithm. The following algorithm specific parameters are used: maximum iterations for each flower plant or population $(M)=200$, function evaluations $=10000$, population size/number of nests $(n)=25$, frequency of updating the loudness and discovery rate of alien eggs/solutions $(\mathrm{Pa})=0.15$. It is observed that the cuckoo search algorithm has obtained optimum value for all the test functions. It is also observed that as the population size is increased, the cuckoo search algorithm reaches to a more optimal solution but converged slowly. This is because of the computational difficulty of CSA.

Both the responses, weld strength and weld width, are optimized, independently. To determine the optimal parametric setting, the developed models, Eqs. (3) \& (4), respectively, are used as objective functions in CSA. Fig. 3 shows algorithm convergence diagrams for CSA with respect to weld strength and weld width. It is worthy to mention here that the statistical variability of the results on repetitive runs is insignificant for CSA which can help to predict the true overall trend of the responses. The average computational times observed for single objective optimization using CSA is 0.315 seconds, which is far less than 1 second.

The results, along with the optimal parameter settings for the responses, are summarized in Table II. The maximum value of weld strength obtained by CSA is $99.47 \mathrm{~N} / \mathrm{mm}$, and the minimum value of weld width obtained is $1.38 \mathrm{~mm}$. The same table also furnishes the results obtained directly by using RSM, in which a search is carried out into the design space to find the local optima using a method, which is known as desirability function analysis (DSA) in response surface methodology. It is found that CSA gives better results than DSA, because CSA searches for global optima and does not keep any parameter at fixed level while searching for the optima.

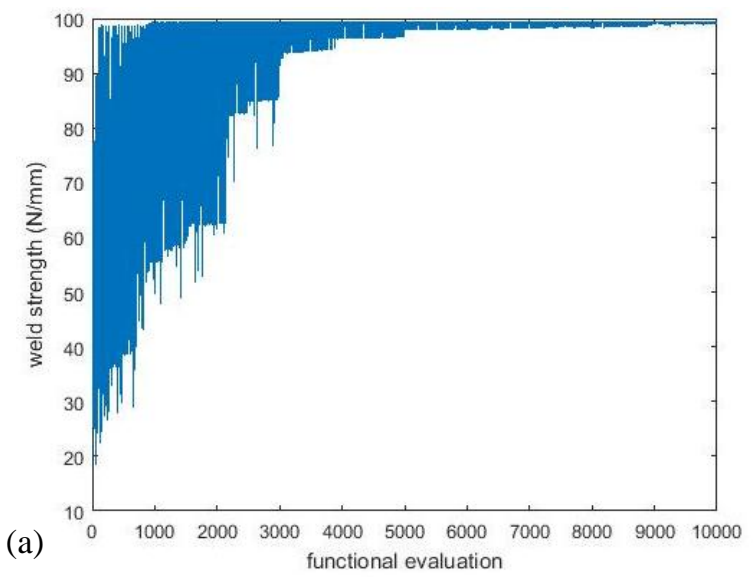




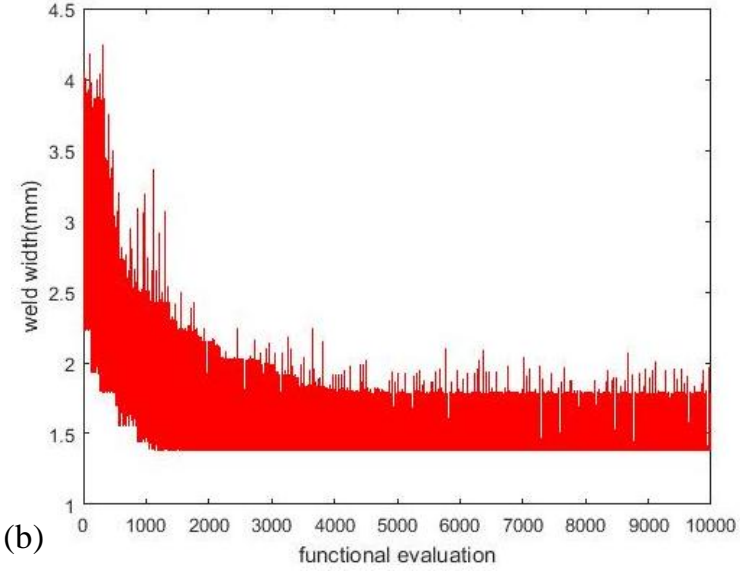

Fig. 3: Algorithm convergence diagrams for (a) weld strength, and (b) weld width using CSA which can resist the larger applied load, thus, increases the weld strength. Clamp pressure ensures good contact between the parts to be welded. This enhances the conduction of heat from the absorptive material to the transparent part and also promotes the molten fluid flow, required for intermixing and cross linking of the polymer chains to combine towards weld formation [1]

It can be depicted from the plots presented in Fig. 5, that the weld width increases with laser power (Fig. 5 (a)) and stand-off distance (Fig. 5 (c)), and decreases with the increases of welding speed (Fig. 5 (b)). Clamp pressure (Fig. 5 (d)) shows insignificant effect on weld width. Increasing the laser power increases the heat input, consequently the width of the weld zone increases. The irradiation time reduces due to the increase of welding speed, which results in a narrow weld. Increasing stand-off distance increases the beam spot size at weld interface, which results in spreading the laser energy onto a wide area, thus increase in weld width.
TABLE II. OPTIMAL RESPONSE VALUES WITH PARAMETRIC SETTINGS

\begin{tabular}{|c|c|c|c|c|c|c|c|}
\hline Response & \multirow{2}{*}{$\begin{array}{c}\text { Nature of } \\
\text { optimization }\end{array}$} & \multirow{2}{*}{$\begin{array}{c}\text { Optimization } \\
\text { Technique }\end{array}$} & \multirow{2}{*}{$\begin{array}{c}\text { Optimal } \\
\text { value }\end{array}$} & \multicolumn{4}{|c|}{ optimal parametric } \\
\cline { 4 - 8 } & & & & $\boldsymbol{P}$ & $\boldsymbol{S}$ & $\boldsymbol{F}$ & $\boldsymbol{C}$ \\
\hline \multirow{2}{*}{ WS } & \multirow{2}{*}{ Maximization } & DFA & 82.26 & 19.84 & 5.01 & 33.82 & 5.33 \\
\cline { 3 - 8 } & & CSA & 99.47 & 20.00 & 4.00 & 37.00 & 3.60 \\
\hline \multirow{2}{*}{ WW } & \multirow{2}{*}{ Minimization } & DFA & 2.09 & 11.73 & 23.23 & 28.44 & 4.27 \\
\cline { 3 - 8 } & & CSA & 1.38 & 10.00 & 16.00 & 25.00 & 6.00 \\
\hline
\end{tabular}

Additional experiments are carried out to check the optimal parametric combination and the response values predicted by the cuckoo search algorithm, and found that the values are in close agreement. Table III furnishes the results of validation test. Any deviation in the result is due to the slight decomposition of the work piece material due to overheating, which the model cannot predict.

TABLE III. VALIDATION OF THE RESULTS

\begin{tabular}{|c|c|c|c|c|c|c|}
\hline \multicolumn{5}{|c|}{ Optimal parametric setting } & Optimal value & \\
\hline & $\boldsymbol{P}$ & $\boldsymbol{S}$ & $\boldsymbol{F}$ & $\boldsymbol{C}$ & & \\
\hline $\begin{array}{c}\text { Weld } \\
\text { strength }\end{array}$ & 20.00 & 4.00 & 37.00 & 3.60 & Predicted & 99.47 \\
\cline { 5 - 7 } & & & & & Experimental & 91.06 \\
\cline { 5 - 6 } & & & & Error (\%) & 9.24 \\
\hline $\begin{array}{c}\text { Weld } \\
\text { width }\end{array}$ & 10.00 & 16.00 & 25.00 & 6.00 & Predicted & 1.38 \\
\cline { 5 - 6 } & & & & & Experimental & 1.51 \\
\cline { 5 - 6 } & & & & Error (\%) & 8.61 \\
\hline
\end{tabular}

\section{ANALYSIS OF PARAMETRIC TRENDS}

Fig. 4 shows the effects of all the process parameters on weld strength. It is apparent from this figure that the laser power (Fig. 4 (a)) and stand-off distance (Fig. 4 (c)) have positive effects and welding speed (Fig. 4 (b)) has a negative effect on the weld strength. Weld strength increases with clamp pressure (Fig. 4 (d)) to its central value and thereafter starts decreasing. Higher laser power increases the heat input and lower welding speed increases the interaction time, thus maximizes the energy delivered at the interaction zone, resulting in increased melt volume and weld strength until the critical temperature of decomposition is reached. Increasing the value of stand-off distance results in larger weld area,
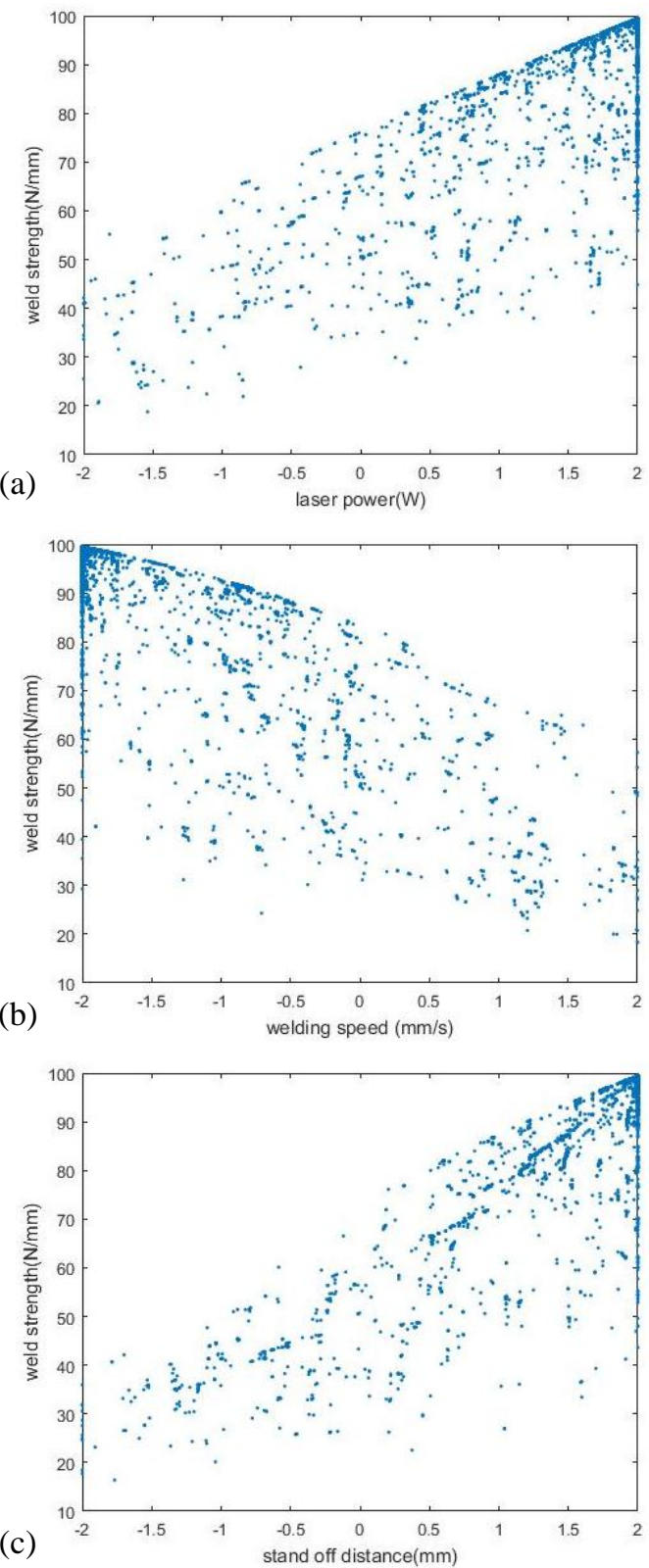


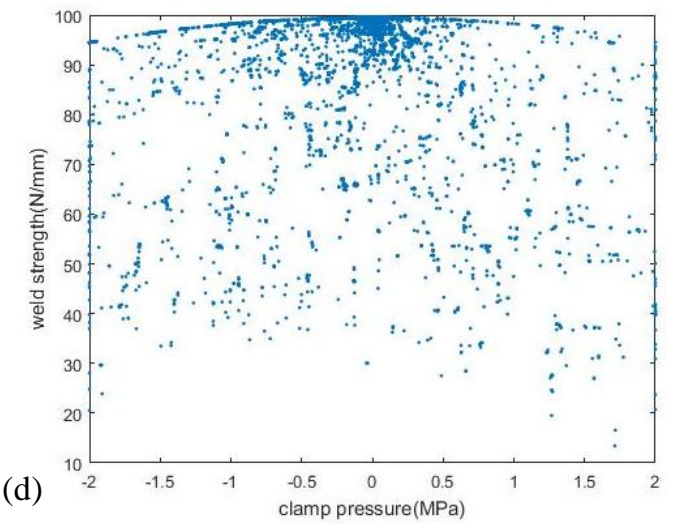

Fig. 4. Scatter diagrams of weld strength for (a) laser power, (b) welding speed, (c) stand-off distance, and (d) clamp pressure
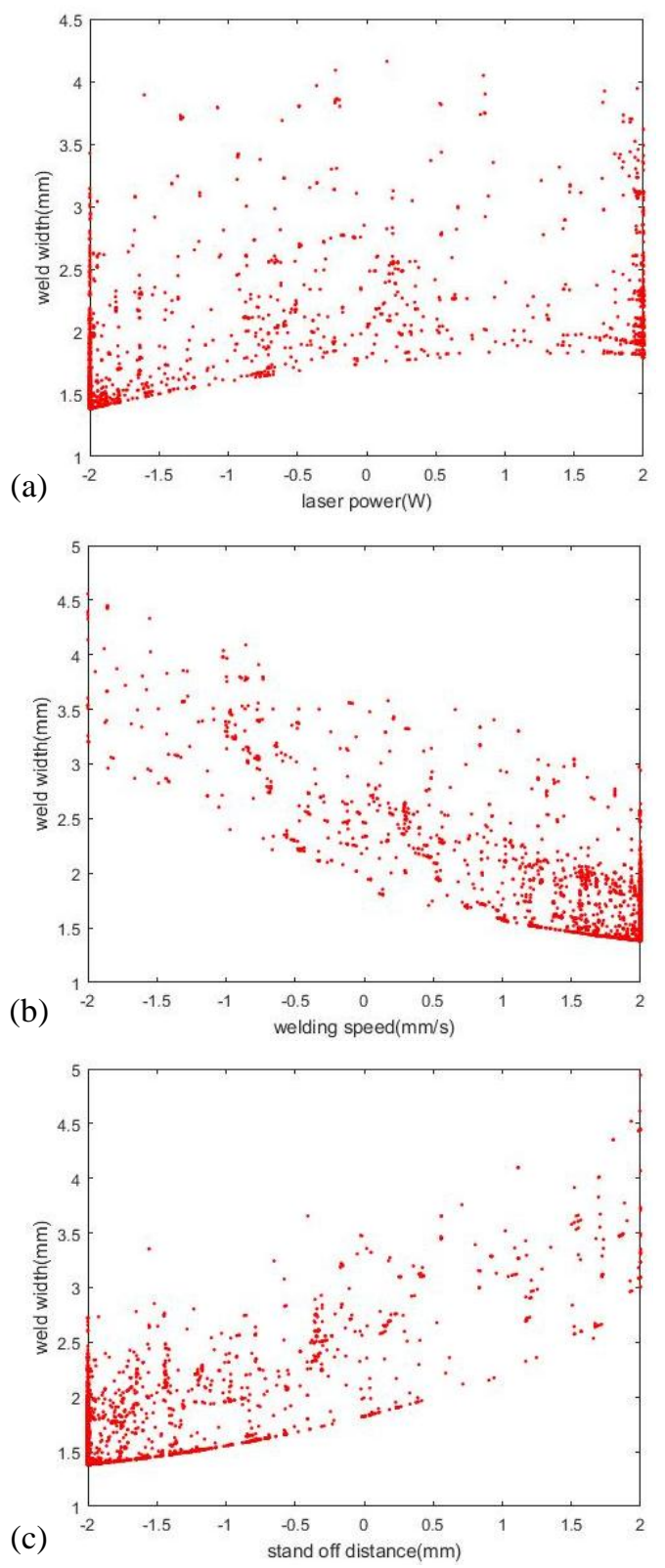

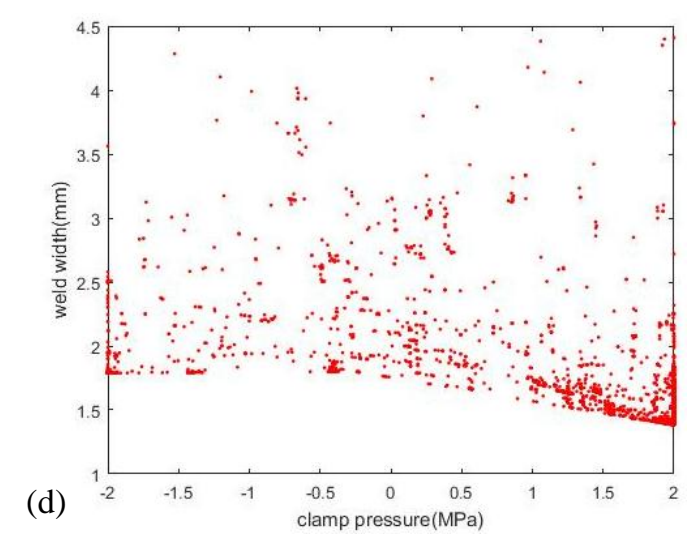

Fig. 5. Scatter diagrams of weld width for (a) laser power, (b) welding speed, (c) stand-off distance, and (d) clamp pressure

\section{CONCLUSION}

Cuckoo search algorithm is successfully implemented for optimization of laser transmission welding parameters and also for prediction of parametric trends. It is seen that CSA has a fast convergence rate and requires very low computational time. The average computational time observed is less than a second. The solutions of the optimization problems show that the algorithm is capable to find the feasible optimal parametric combinations with high degree of accuracy. The results obtained by CSA are compared with those derived directly through RSM based optimization technique, which demonstrates the effectiveness of CSA for optimization of laser transmission welding parameters to enhance the performance characteristics. It is further seen that CSA can predict the true overall parametric trends as it does not require keeping any parameter as constant during the analysis.

\section{References}

[1] B. Acherjee, D. Misra, D. Bose, K. Venkadeshwaran, "Prediction of weld strength and seam width for laser transmission welding of thermoplastic using response surface methodology", Optics and Laser Technology, vol. 41 (8), pp. 956-967, 2009.

[2] X. Wang, C. Zhang, K. Wang, P. Li, Y. Hu, K. Wang, H. Liu, "Multiobjective optimization of laser transmission joining of thermoplastics", Optics \& Laser Technology, vol. 44(8), pp. 2393-2402, 2012.

[3] B. Acherjee, A.S. Kuar, S. Mitra, D. Misra, "Selection of process parameters for optimizing the weld strength in laser transmission welding of acrylics", Procedings of Institute of Mechanical Engineers Part: B - Journal of Engineering Manufacture, vol. 224(10), pp. 15291536, 2010.

[4] J.D. Van de Ven, A.G. Erdman, "Laser Transmission Welding of Thermoplastics - Part I: Temperature and Pressure Modeling”, Journal of Manufacturing Science and Engineering, vol. 129(5), pp. 849-858, 2007.

[5] B. Acherjee, A.S. Kuar, S. Mitra, D. Misra, "Empirical modeling and multi-response optimization of laser transmission welding of polycarbonate to ABS", Lasers in Manufacturing and Materials Processing, vol. 2, pp. 103-123, 2015.

[6] B. Baylis, "Welding thermoplastic elastomers to polypropylene with a diode laser", Proc. of the 21st International Congress on Applications of Lasers \& Electro-Optics, Scottsdale, Arizona, USA, October 14-17, 2002. 


\section{Asia Pacific Journals}

[7] B. Acherjee, S. Mondal, B. Tudu, D. Misra, "Application of artificial neural network for predicting weld quality in laser transmission welding of thermoplastics", Applied Soft Computing, vol. 11(2), pp. 2548-2555, 2011.

[8] D.M.Douglass, C.Y. Wu, "Laser welding of polyolefin elastomers to thermoplastic polyolefin", Proc. of the 22nd International Congress on Applications of Lasers \& Electro-Optics, Jacksonville, Florida, USA, October 13-16, 2003.

[9] R. Prabhakaran, M. Kontopoulou, G. Zak, P.J. Bates, B.K. Baylis, "Contour laser-laser transmission welding of glass reinforced nylon 6", Journal of Thermoplast Composist Materials, vol. 19, pp. 427-439, 2006.

[10] B. Acherjee, A.S. Kuar, S. Mitra, D. Misra, "A sequentially integrated multi-criteria optimization approach applied to laser transmission weld quality enhancement - a case study", International Journal of Advanced Manufacturing Technology, vol. 65(5-8), pp. 641-650, 2013.

[11] B. Acherjee, "Selection of optimal parameters for laser transmission welding of polycarbonate using desirability function analysis", Materials Today: Proceedings, Accepted, 2016.

[12] A.H. Gandomi, A.H. Alavi, X.-S. Yang, "Cuckoo search algorithm: a metaheuristic approach to solve structural optimization problems", Engineering with Computers, vol. 29(1), pp. 17-35, 2013.

[13] A.R. Yildiz, "Cuckoo search algorithm for the selection of optimal machining parameters in milling operations", International Journal of Advanced Manufacturing Technology, vol. 64(1-4), pp. 55-61, 2012.

[14] D.C. Montgomery, "Design and analysis of experiments", New York: Wiley, 2001.

[15] X.S. Yang, S. Deb, "Cuckoo search via Levy flights. Proceedings of World Congress on Nature \& Biologically Inspired Computing (NaBIC), Coimbatore, India, December 9-11, 2009.

[16] X.S. Yang, S. Deb, "Engineering Optimisation by Cuckoo Search", International Journal of Mathematical Modelling and Numerical Optimisation, vol. 1(4), pp. 330-343, 2010. 